\title{
Gene Expression and the Diversity of Identified Neurons
}

\author{
L. BuCK, R. STein, M. PAlazzolo, D.J. ANDERson, AND R. AxeL \\ Institute of Cancer Research, Columbia University, New York, New York 10032
}

Nervous systems consist of diverse populations of neurons that are anatomically and functionally distinct. The diversity of neurons and the precision with which they are interconnected suggest that specific genes or sets of genes are activated in some neurons but not expressed in others. Experimentally, this problem may be considered at two levels. First, what is the total number of genes expressed in the brain, and how are they distributed among the different populations of neurons? Second, can we identify specific genes expressed in individual neurons and relate the expression of these genes to the unique functional properties of these neurons?

A number of investigators have attempted to determine how many genes are expressed in the brain. Recent data suggest that the mRNA population from the entire mammalian brain may be far more diverse than mRNA populations present in homogeneous populations of cells in other, nonneural tissues (Chikaraishi 1979; Van Ness et al. 1979; Chaudhari and Hahn 1983). The brain, however, consists of a large number of heterogeneous cells or cell groupings that are anatomically or biochemically distinct. This immediately raises the question as to whether the complexity of brain mRNA populations is a reflection of a large number of diverse cell types, each expressing a small number of unique neuron-specific sequences. On the other hand, this complexity may result from a novel requirement that all neurons express a significantly greater number of different mRNAs than nonneural cells. The examination of the pattern of gene expression in individual neurons may allow the distinction between these alternatives.

How does one begin to characterize the genes expressed in individual neurons in nervous systems composed of from $10^{2}$ to $10^{12}$ cells? In a direct approach, it is now possible to identify and clone specific gene sequences expressed in individual neurons and attempt to relate the activation of specific genes to the unique functions of individual neurons. A second, indirect approach involves the isolation of genes whose transcription is restricted to the nervous system. The site of expression of these genes can then be explored by in situ hybridization to mRNA in tissue sections through the developing and adult nervous systems.

The simple nervous system of the invertebrate Aplysia californica, a marine snail, is particularly suitable for analyzing specific gene expression because it contains only about 20,000 central nerve cells, which are collected into four pairs of symmetric ganglia and a single asymmetric abdominal ganglion. Moreover, several of the neurons in these ganglia may be recognized by highly reproducible characteristics such as size, shape, position, pigmentation, and function (for review, see Kandel 1979). This numerical simplification has made it possible to relate the function of particular cells to specific patterns of behavior and may permit us to attribute neuronal function to the expression of specific genes. In addition to being few in number, neurons in Aplysia can be quite large, up to $1 \mathrm{~mm}$ in diameter. Most of these large cells are polyploid and contain as much as $2 \mu \mathrm{g}$ of DNA, more than $10^{5}$ times the content of the haploid genome (Coggeshall et al. 1971; Lasek and Dower 1971). Furthermore, our data indicate that mRNA content is proportional to cell size such that the largest of cells contains up to $5 \mathrm{ng}$ of mRNA.

It is therefore possible to dissect out single neurons and examine the activity of individual genes in a single cell. We have constructed both a genomic library from sperm DNA and a library of cDNA clones from the abdominal ganglion of Aplysia and have screened these libraries with probes synthesized from the mRNA of individual neurons. These procedures now permit the identification of genes whose expression is restricted to unique and identified nerve cells of known function. At the limit, with the largest neurons, screening for prevalent sequences can be performed with the cDNA synthesized from a single cell. When these procedures are applied to nerve cells that express prevalent gene products such as peptidergic neurons, clones encoding multiple behavioral neuropeptides can be isolated. In this manner, we have isolated the genes encoding a set of egg laying neuropeptides and, more recently, a gene that encodes a peptide involved in water balance.

\section{Cloning Genes from Clusters of Neurons: The Egg Laying Peptides}

We have previously described a useful experimental system in Aplysia for examining the structure, expression, and modulation of genes that code for a peptide of known behavioral function, the egg laying hormone, ELH (Scheller et al. 1982, 1983a; L.B. McAllister et al., in prep.). The choice of this system for our initial attempts at gene cloning in the Aplysia nervous system was dictated by two considerations. First, ELH is expressed in abundance in an anatomically defined cluster of identical neurons. Second, the neuronal cluster is sufficiently large to permit us to obtain adequate quantities of this mRNA to serve as probe in gene-isolation experiments.

The neuroactive peptide ELH is released from a pair of symmetric and homogeneous clusters of neurons, the 
bag cells, that are attached to the abdominal ganglion (for reviews, see Kandel 1970; Kupfermann 1972; Blankenship 1980; Strumwasser et al. 1980). Each cluster contains about 400 nerve cells, all of which are interconnected electrically and have similar electrophysiologic properties. During the animal's reproductive period, about half of the protein formed in the bag cells is ELH, a 36-amino-acid basic peptide of known sequence (Chiu et al. 1979). The bag cells release several additional peptides along with ELH. The release of these peptides produces a characteristic and stereotyped behavioral repertoire consisting first of a cessation of walking, an inhibition of feeding, and increases in both the respiratory and heart rates, followed by head waving and egg laying.

ELH acts directly on smooth muscle follicles of the ovotestis to initiate egg laying in a manner analogous to the action of oxytocin on myeloepithelial cells in vertebrates (Dudek and Tobe 1979; Rothman et al. 1982). ELH also excites and inhibits identified neurons in the abdominal and buccal ganglia (Branton et al. 1978; Mayeri and Rothman 1982; Stuart and Strumwasser 1980). Thus, ELH is similar in many respects to neuroactive peptides recently characterized in the mammalian brain that act as a neurotransmitter on nerve cells and as a hormone on nonneuronal targets.

We have determined the nucleotide sequence of the ELH gene expressed in the bag cell cluster. The details of the isolation of this gene and its sequence have been described previously (Scheller et al. 1982, 1983a). Here we emphasize a few points that emerge from an examination of the gene sequence. First, the gene containing ELH encodes a 30-kD protein, whereas ELH itself is only $4.4 \mathrm{kD}$. Thus, ELH is synthesized as part of a larger precursor, and its release requires cleavage at pairs of basic residues that flank the 36-amino-acid ELH sequence. The precursor, however, contains several additional pairs of basic residues that may serve as cleavage sites flanking putative neuroactive peptides. A schematic of the protein sequence, along with potential cleavage sites, is shown in Figure 1. Three small pep- tides-the $\alpha$ - and $\beta$-bag cell factors and the acidic peptide-have been isolated from extracts of bag cells and appear to be coordinately released with ELH. Each of these peptides is encoded in the ELH precursor and is bounded by cleavage sites. Furthermore, a role for at least two of these peptides as neurotransmitters altering the activity of specific neurons within the abdominal ganglion has been demonstrated (B.S. Rothman and E. Mayeri, unpubl.).

What are the physiologic functions of these peptides? The entire array of behaviors associated with egg laying can be elicited by excitation of the bag cells. ELH alone, however, cannot elicit the full array of behaviors (S. Mackey and T. Carew; B.S. Rothman and E. Mayeri; both pers. comm.), indicating that additional factors produced by the bag cells are essential in mediating this behavioral repertoire. Our data on the structure of the ELH gene has suggested a mechanism that provides for the coordinate pattern of behaviors associated with egg laying (Scheller et al. 1983). The ELH gene expresses a polyprotein precursor that contains ELH along with several additional neuropeptides. It is therefore tempting to suggest that the behavioral repertoire is mediated by a small set of peptides encoded by a single gene, expressed in a single polyprotein, which is processed and packaged as a unit, facilitating the coordinate release of these peptides. Thus, a single genetic unit may encode the information dictating a complex behavioral repertoire.

Second, the ELH gene is one member of a small multigene family. We have identified two genes, expressed in the atrial gland, that are $90 \%$ homologous to the ELH gene but have diverged at essential sites to generate a unique, nonoverlapping set of peptides. These two genes encode the $\mathrm{A}$ and $\mathrm{B}$ peptides, which themselves are neurotransmitters capable of either directly or indirectly initiating the excitation of the bag cells and the release of ELH. These three members of a multigene family are expressed in a tissue-specific manner. The $A$ and $B$ peptide precursors are synthesized in the atrial gland but not the bag cells, whereas the ELH

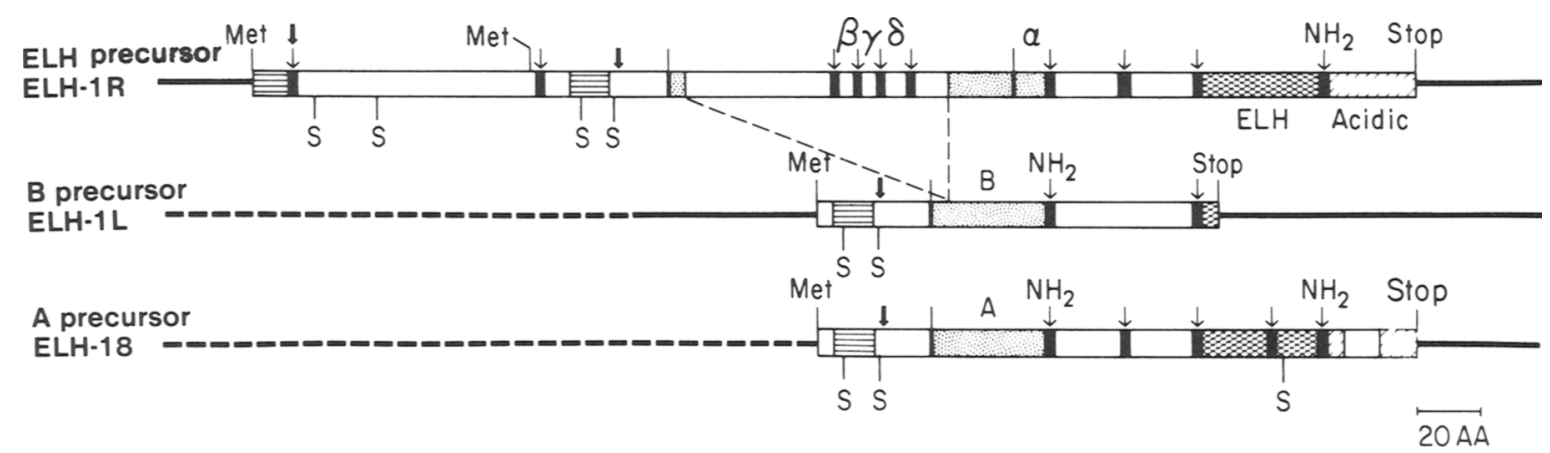

Figure 1. Schematic comparing the precursors encoding the ELH, $\alpha$-, and $\beta$-peptides, deduced from the nucleotide sequences of the peptide genes. Each of the three proteins is initiated by a methionine, followed by a hydrophobic tugion (目). (S) Location of a cysteine residue. ( ) Putative site of cleavage of the signal sequence. Lines above the sequence (|) represent potential cleavages at single arginine residues. ( $\downarrow$ ) Potential or known cleavage at dibasic, tribasic, or tetrabasic residue; if carboxyterminal amidation

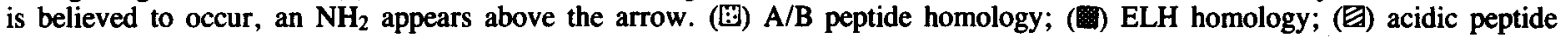
homology. (-) Sequenced, noncoding regions; (----) regions not sequenced. (Reprinted, with permission, from Scheller et al. 1983.) 
precursor is expressed in the bag cells but not the atrial gland.

Finally, we have used in situ hybridization to mRNA in sections through the entire nervous system to ask whether the bag cells and atrial gland are the sole sites of expression of this gene family (L.B. McAllister et al., in prep.). We observe that, in addition to the primary cluster of bag cell neurons expressing the egg laying genes, there exists a network of at least 20 other ELH-producing cells in the small central nervous system of Aplysia. One example of ELH gene expression in two cells among the 2000 cells of the pleural ganglion is shown in Figure 2.

At present we do not understand the physiologic significance of this extensive network of cells. We do not know whether they participate in the egg laying process or whether they carry out completely independent physiologic functions. However, the results indicate that the release of ELH is not restricted to the bag cells. Rather, ELH is synthesized at several additional loci in the Aplysia nervous system. Thus, ELH may serve a more significant role as neurotransmitter than has been previously appreciated.

These three observations serve to illustrate one important point concerning the generation of diversity. A small, multigene family encodes a series of polypro-

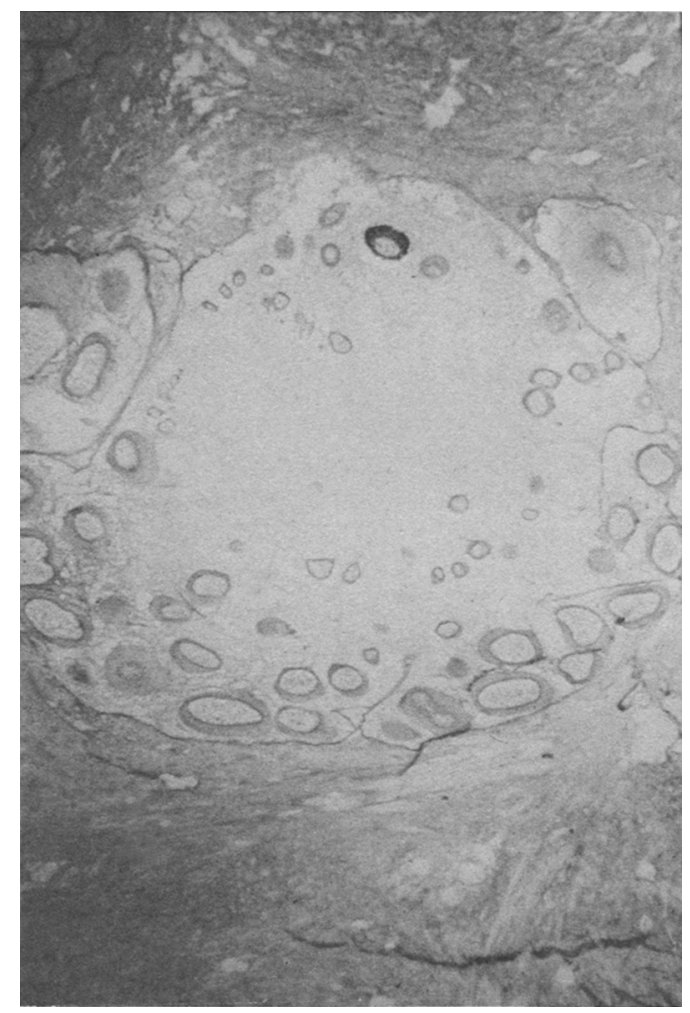

Figure 2. In situ hybridization to mRNA of cells of the pleural ganglion, showing a high density of grains in the cytoplasm of a single neuron. The hybridization probe derives from a clone encoding the ELH gene (Fig. 1) and was nicktranslated to a specific activity of $5 \times 10^{8} \mathrm{cpm}$ with $\left[{ }^{125} \mathrm{I}\right] \mathrm{dCTP}$. teins, each containing the information capable of generating multiple, small neuroactive peptides that are expressed in several different neurons. This multiplicity has suggested combinatorial mechanisms that may be important in generating the diversity observed in the nervous system. The number of possible combinations of egg laying peptides is made even greater by the fact that these peptides are encoded by a small family of genes in which each member has diverged to generate new peptides. Furthermore, these genes encode polyproteins with the ability to generate a large number of additional combinations of peptides merely by altering the pathway of processing in different cell types or in the same cell in response to different stimuli. Furthermore, these peptides are expressed by a network of invariant neurons, suggesting the possibility that they may be used by different cells to effect different functions. The purpose of this diversity might be to activate different patterns of behavior by modulating the activity of different combinations of neurons or target organs.

\section{Cloning Genes Expressed in Individual Neurons: The R15 Peptide}

The egg laying peptides provide an example of the relationship between genes expressed in a cluster of identified neurons and a specific behavior. In Aplysia, physiologic processes may be controlled by single identified neurons. The analysis of the pattern of gene expression in single neurons may therefore permit us to relate the function of individual neurons to the activity of specific genes. We have recently developed procedures that permit the isolation of genes whose expression may be restricted to a single neuron. These procedures have enabled us to clone a gene expressed in a single neuron, $\mathrm{R} 15$, which regulates water balance in Aplysia.

The autonomously bursting neuron, R15 (Fig. 3), synthesizes a protein hormone that, by analogy with the antidiuretic hormone of vertebrates, may control the resorption of $\mathrm{Na}^{+}$(Kupfermann and Weiss 1976). In hypotonic sea water, the animal gains water, which upsets its internal osmolality. Compensating for this effect, the hypotonic sea water is sensed by an organ called the osphradium, which acts via a cluster of sensory neurons to inhibit the burst firing of R15 (JahanParwar et al. 1969; Stinnakre and Tauc 1969). This may inhibit hormone release, diminish $\mathrm{Na}^{+}$resorption, and in this way restore water balance. What is the nature of the peptide released by $R 15$ ? What is its target, and what are its physiologic effects? These questions may be addressed by cloning the gene encoding the R15 peptide, deducing its amino acid sequence, and ultimately synthesizing the peptide to determine its physiologic effects.

In initial experiments, we compared the pattern of proteins synthesized by R15 with those of several other peptidergic neurons within the abdominal ganglion. Abdominal ganglia were exposed to $\left[{ }^{35}\right.$ S] methionine for 24 hours, and individual neurons were isolated and 

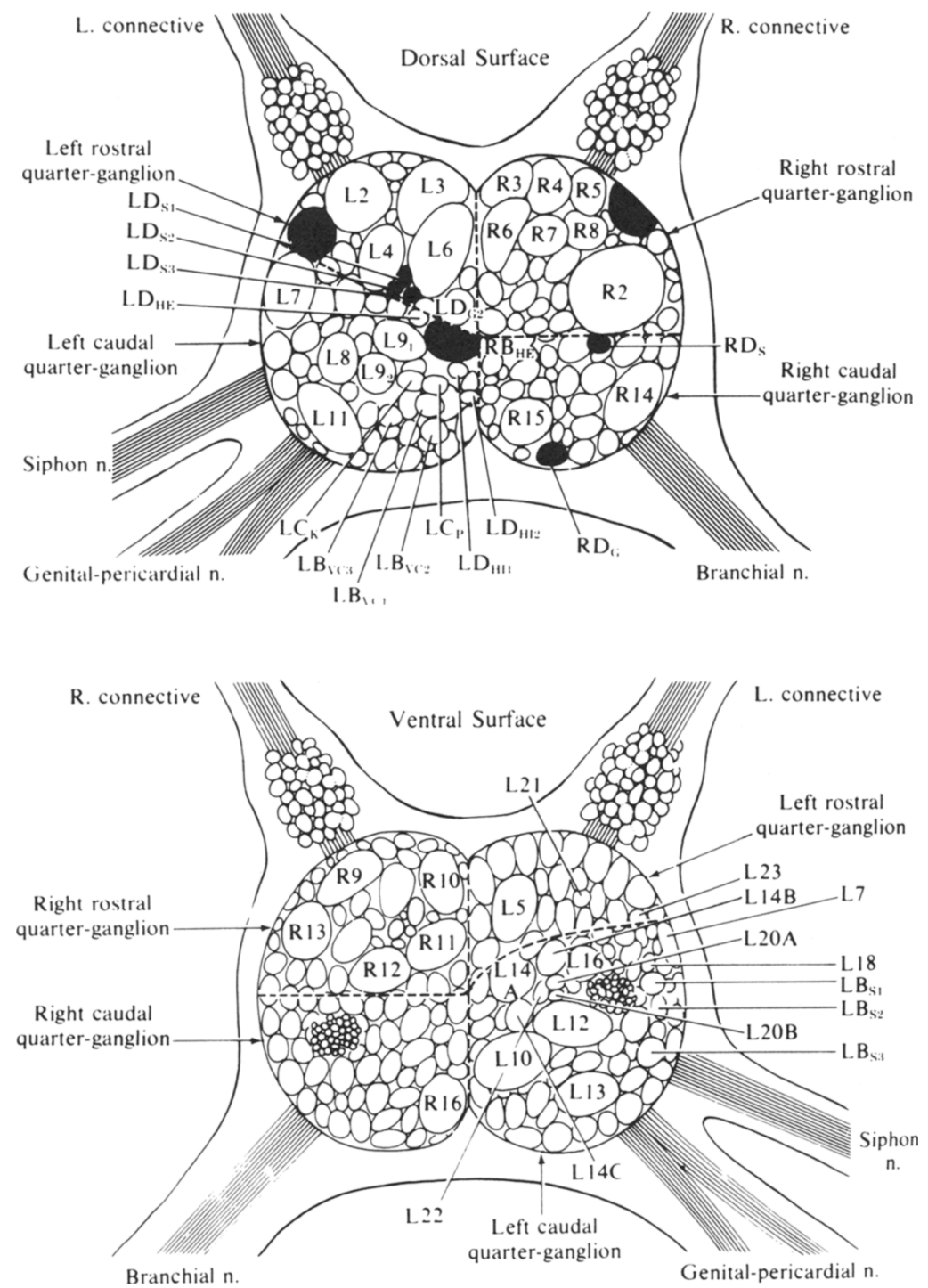

Figure 3. The abdominal ganglion of Aplysia californica. (Reprinted, with permission, from Kandel 1976.)

subjected to SDS-polyacrylamide gel electrophoresis. The autoradiograph shown in Figure 4 shows the patterns of proteins synthesized in single cells. If we concentrate on the low-molecular-weight peptides, we observe that neurons R3 through R14 display identical profiles. The neurons L2, L3, L5, and L6 also appear to comprise a group with a distinct profile, whereas R15, L4, and L11 each possess unique low-molecularweight protein species not present in the other neurons examined. At steady state, the low-molecular-weight peptides of R15 seem to account for only about $5 \%$ of the total proteins synthesized in this cell. Previous studies using shorter labeling periods, however, have demonstrated that about $30 \%$ of the protein in this cell appears as a single, $12.5-\mathrm{kD}$ precursor from which shorter peptides are generated (Loh and Gainer 1975; Strumwasser and Wilson 1976; Aswad 1978). This precursor, thought to contain the water balance peptide, is therefore likely to be encoded by an abundant mRNA.
To isolate the R15 peptide gene, we first constructed a library of cDNA clones from poly (A) ${ }^{+}$mRNA from the entire abdominal ganglion. This library, which consists of $1.3 \times 10^{6}$ independent clones, was constructed utilizing a highly efficient bacteriophage $\lambda$ gt 10 cloning system developed by T. St. John and R. Davis (unpubl.). The neuron R15 comprises about $1 \%$ of the mass of the abdominal ganglion. If the mRNA encoding the water balance peptide comprises $10 \%$ of the mRNA in $\mathrm{R} 15$, then $0.1 \%$ of the mRNA in the ganglion should encode this specific peptide. Thus, 1 out of 1000 clones within the library should contain the peptide sequence. We screened 20,000 independent cDNA clones in duplicate with two highly radioactive cDNA probes, one prepared from the mRNA of R15, which should contain the sequence for the peptide, and one prepared from the cholinergic neuron R2, which should not contain the sequence for the R15 peptide. Both R2 and R15 are large cells, and sufficient cDNA for these experiments can be prepared from as few as five neurons. By comparing 
Figure 4. Analysis of proteins synthesized in individual neurons. Isolated abdominal ganglia were incubated with $\left[{ }^{35}\right.$ S]methionine for $24 \mathrm{hr}$. Single neurons were dissected, solubilized in SDS under reducing conditions, and subjected to SDS-polyacrylamide gel electrophoresis and autoradiography (Laemmli 1970). On the left are shown the sizes $\left(\times 10^{-3}\right)$ of molecularweight standards electrophoresed in an adjacent gel lane. Above each lane is noted the cell in which the proteins were synthesized.

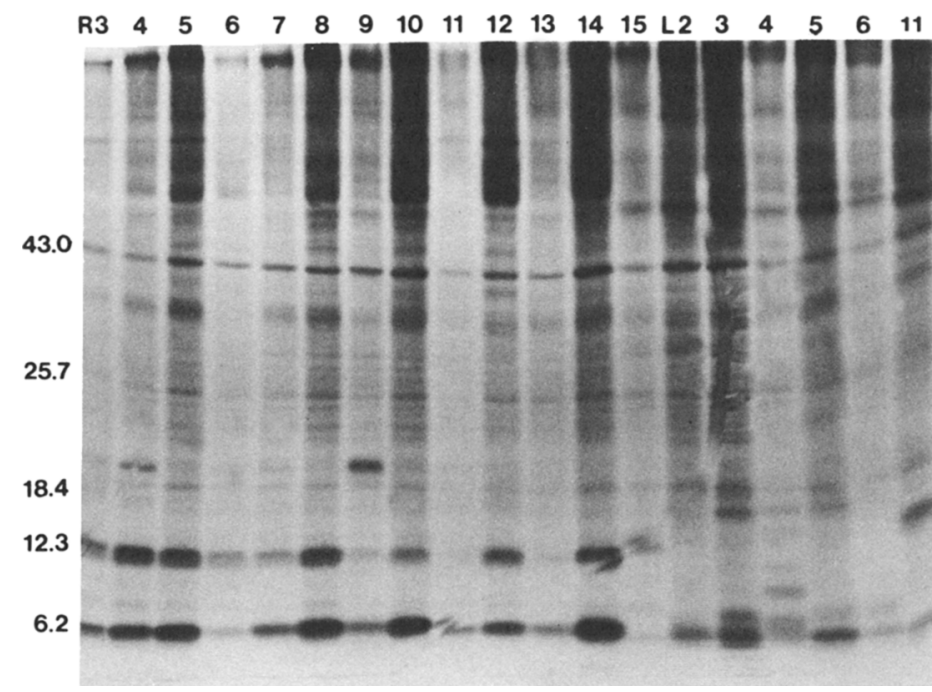

the hybridization of 20,000 individual clones with $R 15$ and R2 cDNA, we were able to isolate five clones that reproducibly hybridize with cDNA from R15 but not $R 2$. An example of the results of this differential screen are shown in Figure 5.

R15-specific clones were picked, plaque-purified, and the DNAs were characterized by restriction endonuclease mapping. All five clones contain identical sequences but extend variable distances at their $5^{\prime}$ termini. The variability in length results from incomplete reverse transcription during cDNA synthesis. The largest of these five clones is 700 nucleotides in length. We utilized this clone as a probe in Northern blotting experiments to determine the size of the mRNA from which this clone was originally synthesized. Northern blotting to mRNA of the abdominal ganglion reveals a single hybridizing species $3.5 \mathrm{~kb}$ in length (Fig. 6). We therefore rescreened the library and obtained a $3.5-\mathrm{kb}$ clone, which is likely to be full length.

The frequency with which this sequence is identified in differential screens suggests that it is an abundant sequence in neuron R15 but is not present in abundance in neuron R2. Dot-blotting experiments further suggest that this sequence is not expressed in any other neuron within the abdominal ganglion. RNA was prepared from neurons $R 15, R 14$, and $R 2$, and collections of right- and left-upper-quadrant peptidergic neurons. Hybridization with the R15 clone is only observed with RNA from neuron R15 (Fig. 7). In a separate experiment, we observed no hybridization to RNA from the entire abdominal ganglion from which neuron R15 had been removed.

As a control, we have also isolated a cDNA clone that hybridizes strongly to RNA from the right-upperquadrant neurons (R3-R8) as well as from neuron R14 but shows no hybridization to RNA from R2, R15, or the left-upper-quadrant cluster (Fig. 7). This clone is likely to encode the abundant set of low-molecularweight proteins shared by neurons R3-R14 (Fig. 4) and may encode a cardioactive peptide characterized in detail by Scheller et al. (1983b).
The unique cell-specific pattern of expression of the R15 clone, together with its abundance in R15 mRNA populations, suggests that it encodes the major water balance peptide. However, this is not yet proven. The sequence of this peptide is unknown and antibodies are unavailable. We are currently determining the nucleotide sequence of the 3.5-kb cloned DNA. From this sequence we may deduce the amino acid sequence of the protein precursor and the smaller peptides contained within it. The peptides may then be synthesized and their physiologic activities determined.

\section{Gene Diversity: Rare and Abundant Genes}

Recent data suggest that the mRNA population from the entire mammalian brain may be far more diverse than the mRNA populations present within homogeneous populations of cells in other tissues. The abundance of these brain-specific RNAs suggests two extreme models of their distribution: Either all brain mRNA species are equally represented at low frequency in all neurons, or they are present in small populations of neurons in high abundance. By examining the pattern of gene expression in individual, identified neurons, we can directly test the second of these two alternatives.

We have compared the genes expressed in a set of three cholinergic neurons in the abdominal ganglion, $R 2$, L11, and L10, by the differential screening procedure described earlier. This allows an initial estimate of the diversity among moderately prevalent sequences in functionally distinct neurons sharing the same classical transmitter. For example, R2 is a giant cell that comprises $5 \%$ of the mass of the ganglion. Thus, in a randomly selected collection of 50,000 cDNA clones from the abdominal ganglion library, $5 \%$ or 2500 would be expected to derive from R2. If $R 2$ did in fact contain a unique message present at a level of about $1 \%$, one would expect that 25 clones would be present among the 50,000 , which would give a differential signal when compared with probe from L11 and L10 

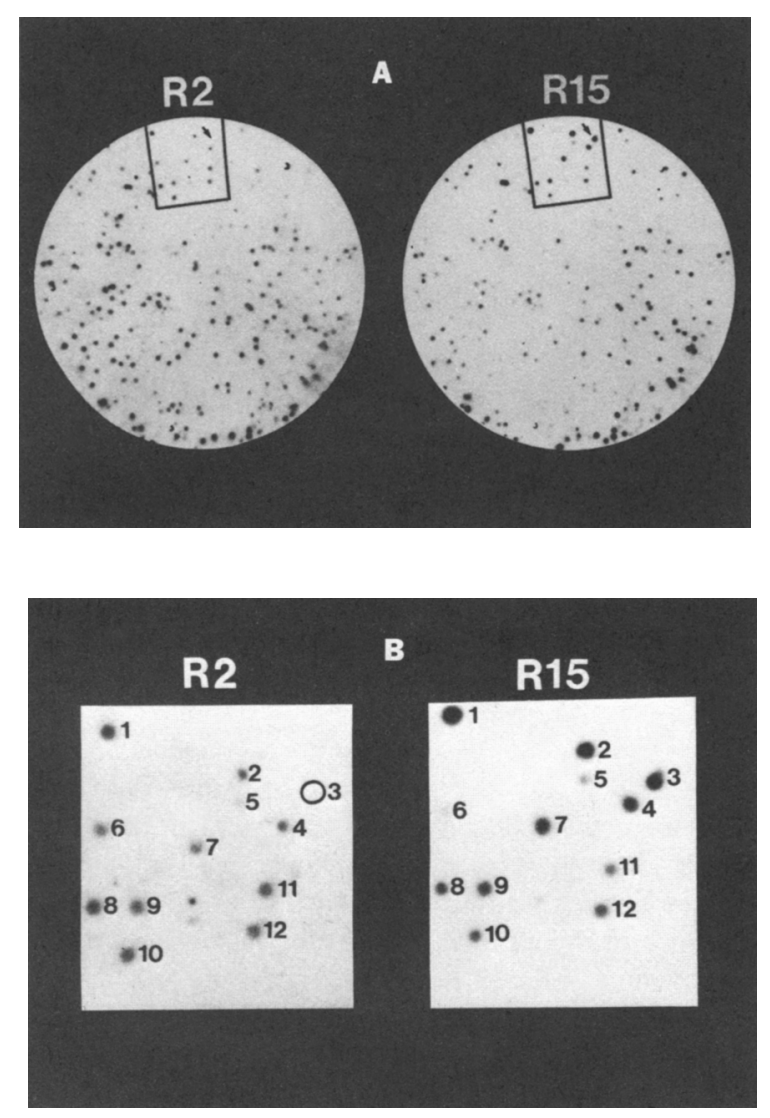

Figure 5. Isolation of neuron-specific cDNA clones by differential screening. Recombinant phage from the abdominal ganglion cDNA library were plated at low density and screened. Replicate filters were hybridized with ${ }^{32} \mathrm{P}$-labeled cDNA prepared from R2 and R15 RNA by oligo(dT)-primed reverse transcription. Shown are autoradiographs of the replicate filters following hybridization $(A)$. The boxed areas are enlarged below $(B)$. $(\rightarrow)$ Location of a R15 peptide clone that hybridized to cDNA prepared from R15, but not R2, RNA.

mRNA. Moreover, if $\mathbf{R} 2$ possessed more than one unique, but abundant transcript, many times more than 25 such clones would be detected.

In preliminary experiments, we have observed transcripts that do distinguish R2 and L11 from one another. In screening 50,000 clones from the abdominal ganglion library, of which $50-60 \%$ reacted with our cDNA probes, we found one clone expressed in R2 but not L11 and another expressed in the latter neuron but not the former. In addition, we found a second clone expressed at much higher levels in R2 than in L11. We estimate that these clones represent mRNAs present at a level of about $0.1-2.0 \%$ of the total, so that they are moderately abundant species. Although these mRNAs show a clear differential distribution between the cells from which the cDNA probes were made, preliminary results suggest they may be present in other neurons in the ganglia as well.

Thus, preliminary studies of a small number of cells suggest that the diversity among individual neurons and the apparent complexity ascribed to neuronal mRNA

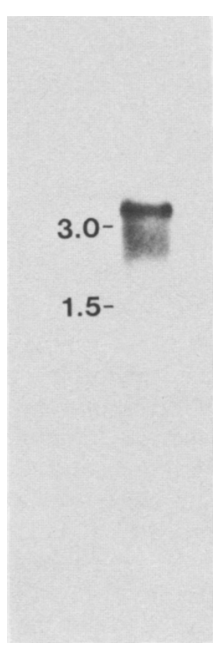

Figure 6. Determination of the size of the mRNA encoding the R15 peptide. Abdominal ganglion poly(A) ${ }^{+}$RNA was electrophoresed, blotted onto nitrocellulose, and hybridized with the cloned R15-specific cDNA labeled by nick translation. Sizes shown on the left (kb) indicate the migration of $E$. coli rRNA molecules coelectrophoresed with the ganglion RNA.

may be reflected in the differential synthesis of relatively abundant mRNAs in different neurons sharing the same transmitter. Furthermore, the detection of quantitative differences in the level of certain mRNAs between cholinergic neurons suggests that neuronal diversity may in part be explained by overlapping sets of gene products differentially expressed in different cells. This "combinatorial" model represents an intermediate case between the two extreme models of mRNA distribution discussed earlier. We emphasize, however, that our annealing conditions will only allow us to detect clones expressed at a level of $0.1 \%$ or higher in the cell from which the cDNA was prepared. It is still possible that Aplysia neurons are further distinguished by qualitative differences in the expression of relatively low-abundance genes. To test this possibility, it will be necessary to construct cDNA libraries from individual neurons, permitting the detection of low-level RNA transcripts, which would be present at vanishingly low frequencies in a library constructed from the entire ganglion.

\section{CONCLUSIONS}

We have examined genes expressed in single neurons of the mollusc Aplysia and have attempted to relate the expression of specific genes to the function of individual neurons. From an analysis of genes active in a small number of cells, it is also possible to make some preliminary statements on the pattern of gene expression in the nervous system as a whole.

First, are unique genes expressed in specific neurons? The peptidergic neuron R15 expresses a pre- 


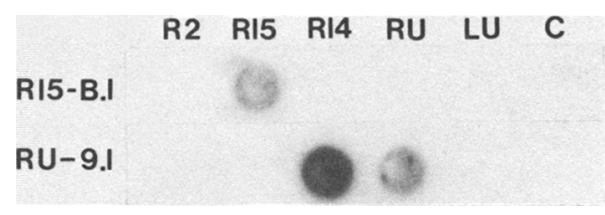

Figure 7. Tissue-specific expression of the peptide genes. RNAs extracted from two R2, two R15, eight R3-8 (RU), and eight L2, L3, L4, L6 (LU) neurons, and $E$. coli tRNA (C) were dot-blotted onto nitrocellulose and hybridized with ${ }^{32} \mathrm{P}$ labeled cloned cDNA encoding the R15 (R15-B.1) or R14 (RU-9.1) peptide.

dominant mRNA thought to encode a peptide regulating $\mathrm{Na}^{+}$and water balance. At current levels of detection, the gene encoding this mRNA is neuron specific; it is expressed in R15 but not in any other neurons of the abdominal ganglion. In addition to this neuron-specific gene, we have isolated a gene whose expression appears to be restricted to a small group of neurons within the ganglion. This gene, which may encode a cardioactive peptide, is expressed in R14 and also expressed in the peptidergic cells clustered in the right-upper quadrant of the ganglion. An even more extensive distribution is observed for the expression of the family of genes encoding the egg laying peptides. At least one member of this small, multigene family is expressed in the bag cell cluster, the atrial gland, and a network of at least 20 individual neurons expressed in four of the five ganglia of the nervous system.

What may the functional significance of these diverse tissue-specific patterns of gene expression be? In a cluster of identical neurons, such as the bag cells, in which the cells are electrically interconnected, a single excitation causes each cell in the cluster to fire and release an identical collection of gene products. Thus, a cluster of identical cells may be required, as in other organs, to meet the quantitative demands for a specific gene product, which in this case is a set of peptides. Anatomically dispersed cells expressing common gene products, such as the ELH-producing cells distributed throughout four ganglia as well as R14 and the rightupper-quadrant neurons expressing the cardioactive peptide, may function quite differently. If these dispersed cells receive different input, then the same peptide may be released at different times by different cells in several different anatomic locations, perhaps in combination with different companion peptides, to effect several distinct physiologic or behavioral processes.

What do we learn about the function of specific neurons from an analysis of the genes they express? From the nucleotide sequence of the ELH gene, we have demonstrated that this gene encodes not a single peptide, ELH, but several additional neuroactive peptides released from the bag cells that are active as neurotransmitters on cells of the abdominal ganglion. We have previously suggested that the entire repertoire of egg laying behaviors may be mediated by a set of neuropeptides encoded by a single gene. We anticipate that the nucleotide sequence of the R15 peptide will allow us to explore the physiologic processes involved in water balance since at present the amino acid sequence of the active peptide is unknown and its cellular targets or mode of action have not been determined.

What seems to be emerging from the analysis of peptidergic neurons and peptide genes in Aplysia is that neurons may use combinatorial mechanisms at several levels to account, at least in part, for the diversity required in the nervous system. The existence of multiple genes that encode polyproteins containing several neuroactive peptides expressed in diverse cells, if exploited, could greatly expand the informational content of the genome.

The peptidergic neurons expressing ELH, the cardioactive peptide, and the R15 peptide are distinguished by the expression of unique, prevalent mRNA species encoding different peptides. A different pattern is emerging from the analysis of functionally distinct neurons that share the transmitter acetylcholine. In preliminary experiments we have detected prevalent transcripts expressed in different cholinergic cells. These transcripts, however, appear to be expressed in other neurons in the abdominal ganglion. The diversity of these neurons may therefore result not from the expression of genes unique to individual cells, but from unique but overlapping patterns of gene expression in functionally distinct neurons.

\section{ACKNOWLEDGMENTS}

This work was supported by a grant from the $\mathrm{Na}$ tional Institutes of Health to R.A. and by fellowships from the Cancer Research Institute, Inc., to L.B., from the National Institutes of Health to R.S., and from the Helen Hay Whitney Foundation to D.A.

\section{REFERENCES}

AswAD, D.W. 1978. Biosynthesis and processing of presumed neurosecretory proteins in single identified neurons of Aplysia californica. J. Neurobiol. 9: 267.

Blankenship, J.E. 1980. Physiological properties of peptidesecreting neuroendocrine cells in the marine mollusc Aplysia. In The role of peptides in neuronal function (ed. J.L. Barker and T.G. Smith, Jr.), p. 160. Marcel Dekker, New York.

Branton, W.D., E. Mayeri, P. Brownell, and S. Simon. 1978. Evidence for local hormonal communication between neurons in Aplysia. Nature 274: 70.

Chaudhari, N. and W.E. HahN. 1983. Genetic expression in the developing brain. Science 220: 924 .

Chikaraishi, D.M. 1979. Complexity of cytoplasmic polyadenylated and nonpolyadenylated rat brain ribonucleic acids. Biochemistry 18: 3249.

Chiu, A.Y., M.W. Hunkapiller, E. Heller, D.K. Stuart, L.E. Hood, and F. STrumwasser. 1979. Neuropeptide egg-laying hormone of Aplysia californica: Purification and primary structure. Proc. Natl. Acad. Sci. 76: 6656 .

Coggeshall, R.E., B.A. YaKSTA, and F.J. Swartz. 1971. A cytophotometric analysis of the DNA in the nucleus of the giant cell, R-2, in Aplysia. Chromosoma 32: 205.

DudEK, F.E. and S.S. TOBE. 1979. Bag cell peptides act 
directly on ovotestis of Aplysia californica: Basis for an in vivo bioassay. Gen. Comp. Endocrinol. 36: 618.

Jahan-Parwar, B., M. Smith, and R. Von Baumgarten. 1969. Activation of neurosecretory cells in Aplysia by osphradial stimulation. Am. J. Physiol. 216: 1246.

KANDEL, E.R. 1970. The organization of subpopulations in the abdominal ganglion of Aplysia. In The interneuron (ed. A. Scheibel), p. 71. UCLA Press, Los Angeles.

- 1976. Cellular basis of behavior: An introduction to behavioral neurobiology. W.H. Freeman, San Francisco. . 1979. Behavioral biology of Aplysia. W.H. Freeman, San Francisco.

KUPFERMANN, I. 1972. Studies on the neurosecretory control of egg laying in Aplysia. Am. Zool. 12: 513.

KUPFERMANN, I. and K.R. WEIss. 1976. Water regulation by a presumptive hormone contained in identified neurosecretory cell R15 of Aplysia. J. Gen. Physiol. 67: 113. from individual Aplysia neurons: Neuropeptide modulators of cardiovascular physiology. Cell (in press).

LAEMMLI, U.K. 1970. Cleavage of structural proteins during the assembly of the head of bacteriophage T4. Nature 227: 680 .

LASEK, R.J. and W.J. DowER. 1971. Aplysia californica: Analysis of nuclear DNA in individual nuclei of giant neurons. Science 172: 278.

LoH, Y.P. and H. GaINER. 1975. Low molecular weight specific proteins in identified molluscan neurons. II. Processing, turnover and transport. Brain Res. 92: 193.

MAYERI, E. and B. ROTHMAN. 1982. Nonsynaptic peptidergic neurotransmission in the abdominal ganglion of Aplysia. In Neurosecretion-Molecules, cells and systems (ed. D.S.
Farner and K. Lederis), p. 307. Plenum Press, New York. Rothman, B.S., G. Weir, and F.E. Dudek. 1982. Direct action of egg-laying hormone on ovotestis of Aplysia. Science 197: 490 .

Scheller, R.H., J.F. Jackson, L.B. McAllister, B.S. Rothman, E. Mayeri, and R. Axel. 1983a. A single gene encodes multiple neuropeptides mediating a stereotyped behavior. Cell 32: 7.

Scheller, R.H., J.R. NAmbu, R. TAussig, and A.C. MAHON. 1983b. Isolation of genes using cDNA probes

SCHELler, R.H., J.F. JACKSON, L.B. MCAllister, J.H. Schwartz, E.R. Kandel, and R. AXel. 1982. A family of genes that codes for ELH, a neuropeptide eliciting a stereotyped pattern of behavior in Aplysia. Cell 28: 707.

Stinnakre, J. and L. Tauc. 1969. Central neuronal response to the activation of osmoreceptors in the osphradium of Aplysia. J. Exp. Biol. 51: 347.

Strumwasser, F. and D.L. Wilson. 1976. Patterns of proteins synthesized in the R15 neuron of Aplysia. Temporal studies and evidence for processing. J. Gen. Physiol. 67: 691 .

Strumwasser, F., L.K. Kaczmarek, A.Y. ChiU, E. Heller, K.R. Jennings, and D.P. Viele. 1980. Peptides controlling behavior in Aplysia. Soc. Gen. Physiol. Ser. 35: 197.

StUarT, D.K. and F. Strumwasser. 1980. Neuronal sites of action of a neurosecretory peptide, egg-laying hormone, in Aplysia californica. J. Neurophysiol. 43: 399.

VAN Ness, J., I.H. MaXWELL, and W.E. HahN. 1979. Complex population of nonadenylated messenger RNA in mouse brain. Cell 18: 1341. 


\section{$\$_{\text {CSH }}^{\infty} \mathrm{C}$ Cold Spring Harbor Symposia SYMPOSIA}

\section{Gene Expression and the Diversity of Identified Neurons}

L. Buck, R. Stein, M. Palazzolo, et al.

Cold Spring Harb Symp Quant Biol 1983 48: 485-492

Access the most recent version at doi:10.1101/SQB.1983.048.01.053

References This article cites 21 articles, 7 of which can be accessed free at:

http://symposium.cshlp.org/content/48/485.refs.html

Email alerting Receive free email alerts when new articles cite this article service sign up in the box at the top right corner of the article or click here

To subscribe to Cold Spring Harbor Symposia on Quantitative Biology go to: http://symposium.cshlp.org/subscriptions 